\title{
Kant plantenn, kant anv. Inflorescence linguistique en Basse-Bretagne
}

Kant plantenn, kant anv: linguistic inflorescence in Lower Brittany

Laurent Gall

\section{(2) OpenEdition}

\section{Journals}

Édition électronique

URL : https://journals.openedition.org/lbl/1017

DOI : $10.4000 / \mathrm{lbl} .1017$

ISSN : 2727-9383

Éditeur

Université de Bretagne Occidentale - UBO

\section{Édition imprimée}

Date de publication : 1 juin 2015

Pagination : $7-25$

ISBN : 979-10-92331-16-5

ISSN : 1270-2412

\section{Référence électronique}

Laurent Gall, «Kant plantenn, kant anv. Inflorescence linguistique en Basse-Bretagne », La Bretagne Linguistique [En ligne], 19 | 2015, mis en ligne le 01 mai 2021, consulté le 22 mai 2021. URL : http:// journals.openedition.org/lbl/1017 ; DOl : https://doi.org/10.4000/lbl.1017

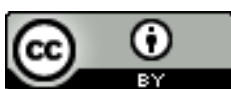

La Bretagne Linguistique est mise à disposition selon les termes de la Licence Creative Commons Attribution 4.0 International. 


\title{
Kant plantenn, kant anv. Inflorescence linguistique en Basse-Bretagne
}

\begin{abstract}
D。
es enquêtes ethnographiques de terrain, réalisées pour l'essentiel en centre Bretagne ${ }^{1}$, délivrent un recueil d'usages et de dénominations de plantes. On peut ainsi mettre à jour les types motivationnels à l'œuvre pour les divers phytonymes : propriétés sensitives mises en jeux, traits morphologiques et anatomiques, analogie entre végétaux ou avec des organes d'animaux, couleurs, nombres, propriétés (mécaniques, thérapeutiques...), connotations religieuses, etc. L'analyse lexicale dégage de cette première étape de travail certains axes fondamentaux de l'interprétation vernaculaire du monde végétal.

L'érosion simultanée des savoirs populaires et de la langue bretonne entraîne une raréfaction des personnes détentrices de connaissances fines en breton sur les êtres vivants et les écosystèmes. Ces gisements d'ethno-savoirs ont été plus fournis, plus diversifiés
\end{abstract}

* Doctorant en ethnologie, CRBC (EA 4451-UMS 3554), UBO/ueb.

1. Les informations sont recueillies depuis 2002 auprès de locuteurs bretonnants natifs du centre Bretagne ; le terrain est circonscrit à huit communes du sudouest des Côtes d'Armor, localisées sur le massif de Quintin : Bulat-Pestivien, Saint-Servais, Saint-Nicodème, Trémargat, Lanrivain, Kérien, Peumerit Quintin, Maël Pestivien. 
et mieux répartis dans les sociétés passées où la considération du milieu naturel était partie intégrante de la vie quotidienne. L'appauvrissement d'une ressource ne signifie pas pour autant pénurie totale et n'interdit pas d'analyser les données en vue d'interpréter la classification populaire du végétal. Hypothétique classification car la nomenclature dont les pièces sont plus dispersées et usées que jamais, est ici reconstituée, à la façon d'un puzzle.

Alors quelles pièces de la flore nomment les indigènes dont le regard ne s'est pas définitivement détourné de la nature ? Nommet-on uniquement pour des raisons matérialistes les plantes utiles et celles à la présence intempestive, que l'on doit connaître pour mieux les éliminer? Nomme-t-on aussi pour des raisons plus immatérielles, celles aux caractères floraux qui font signe dans la haie et le bosquet (la primevère, boked an hañv «fleur de l'été» annonciatrice des beaux jours ; l'aubépine, spern gwenn, au fleurissement d'une blancheur virginale du mois de mai, «mois de Marie»...) ? Nomme-t-on en raison d'une perception sensitive de la flore où le goût astringent de la prunelle, le parfum du chèvrefeuille à la tombée de la nuit, le fruit de la bardane qui s'agrippe aux vêtements forment autant de repères stimulant les sens au creux des chemins parcourus par les savoirs buissonniers?

L'effort de reconstitution du puzzle phytonymique solliciterait, d'une part, un questionnement sociolinguistique... qui ne sera pas défloré ici. Aux questions du «comment nomme-t-on», on peut surenchérir par «qui parle» - encore - de la flore? Face aux significations inscrites dans les divers niveaux sémantiques portés par l'expression orale, on pourrait aussi interroger «ce que parler de la flore veut dire ", pour reprendre l'intitulé de l'ouvrage de Pierre Bourdieu ${ }^{2}$, puisque s'exprimer sur la nature et les plantes est bien souvent un artifice ou un jeu verbal pour parler des humains. Comment les manières de désigner les plantes sont-elles partagées entre les registres français et breton ? Certaines sont exclusivement nommées en breton, d'autres « ne connaissent pas » le breton.

2. Pierre Bourdieu, Ce que parler veut dire. L'économie des échanges linguistiques, Paris, Fayard, 1982. 
Et puis savoir nommer, est-ce savoir utiliser? Le savoir-dire et le savoir-faire vont-ils de pair? L'écart entre le geste et la parole ne cesse de grandir, d'autant que la mémoire collective a été rendue inaccessible pour cause «d'obsolescence programmée». L'anthropologue qui enquête aux confins de la mémoire sait la barrière à passer avant d'accéder à des savoirs distillés, au compte-gouttes. L'alambic est parfois en panne, d'autrefois il marche du feu de Dieu ! Le savoir est précieux... comme vieilli en fût de chêne.

\section{De la variabilité du lexique pour nommer les plantes}

Pour combler une béance dans un monde sans nom et inmaîtrisable, la première opération de l'esprit humain est de nommer les êtres vivants afin de mettre fin au désordre naturel, de le rendre intelligible et de pouvoir s'en approprier les ressources.

Classification universelle de la flore, la botanique scientifique attribue une place unique à chaque espèce. Là où le botaniste attribue un seul nom, le praticien indigène dispose d'une série de noms à la forte variabilité géographique pour désigner une espèce ou un groupe d'espèces. Exemple de synonymie vernaculaire, le genre Plantago, le plantain, peut être désigné par au moins onze phytonymes différents $^{3}$. Au sein de ce même taxon, de nombreux locuteurs distinguent plus précisément deux sous-taxons populaires et savent nommer les deux espèces les plus fréquentes, plantain majeur, Plantago major, et plantain lancéolé, Plantago lanceolata.

Principe inverse de la synonymie, le recueil des noms de plantes sur le terrain tient également compte des nombreuses homonymies : différentes espèces, parfois apparentées, parfois pas du tout, peuvent porter le même nom. C'est le cas de stankerez ar gwad «celle qui bouche le sang» pour qualifier à la fois plantain(s) et achillée millefeuille.

La distribution géographique ne subit pas les mêmes variations en tout lieu et fluctue selon les catégories de plantes. Une série de noms de plantes présente une forme lexicale relativement stable sur

3. Données personnelles issues du terrain. 
le territoire de la Basse-Bretagne. Dans cet ensemble, on trouve les noms d'arbres (châtaignier, kistin ; chêne derw ; hêtre, faou...), certains arbustes (ajonc, lann; aubépine, spern gwen ; prunellier, spern $d u \ldots$ ), arbrisseaux (bruyère, brug), certaines herbacées sauvages (ortie, linad ; ronce, drez...) et les plantes de cultures (céréale, ed; avoine, kerc'h ; choux, kaol...). Les noms les plus stables sont ceux des plantes que l'on sait communément distinguer et nommer, quel que soit le niveau de connaissance en botanique : chêne, châtaignier, ronce et ortie comptent parmi les plantes les mieux identifiées au sein d'une population. D'autres végétaux, en revanche, se caractérisent par une fluctuation importante de leur construction lexicale : il s'agit principalement de plantes herbacées sauvages. La variabilité touche davantage les plantes les moins socialisées, celles dont on use peu souvent (considération d'ordre matériel) ou celles que l'on perçoit moins parce que moins répandues et de petite taille (considération d'ordre cognitif).

\section{Cent plantes et cent manières de les nommer}

Kant bro, kant kis

Kant parrez, kant iliz

«Cent pays, cent modes, cent paroisses, cent églises » : le dicton qui décrit la diversité culturelle du pays et de ses terroirs reflète tout autant la richesse linguistique contenue dans les noms de plantes en Basse-Bretagne. Prolongeons-le par kant plantenn, kant anv «cent plantes et cent noms» (si ce n'est mille). L'imaginaire tourne à plein pour qualifier le monde végétal et mobilise ses tournures d'esprit créatrices, où l'utile se mêle à la poésie et l'agréable dans le but d'identifier, de classer et de mémoriser la diversité de la nature. L'effort d'ornementation de la langue que l'on ne retrouve pas toujours de manière aussi prononcée dans d'autres domaines de la vie est révélateur de l'essence inspiratrice du végétal, toutes sociétés confondues.

La façon d'apposer un nom sur les plantes ne marche pas comme une girouette exposée au vent, sans direction cardinale logique. L'art d'attribuer un nom est codé par le cadre culturel, par le milieu social et technique, bref par la façon dont la société envisage le compagnonnage avec son environnement végétal. 
Selon C. Friedberg, anthropologue des ethnosciences, le processus classificatoire emprunte trois types d'opérations logiques : l'identification, la dénomination, l'insertion dans un système de référence. Elle estime que

«La reconnaissance de la diversité des êtres vivants et leur classification constituent deux niveaux d'organisation des savoirs. Il en existe un troisième englobant l'ensemble des êtres vivants, y compris les êtres humains, dans le fonctionnement de chaque société. [...] L'idée que l'on se fait des êtres vivants en tant qu'individus et de leur insertion dans un système organisé est subordonnée au concept d'unité et de continuité du vivant ${ }^{4}$. »

La présentation d'une typologie des motivations lexicales au sein de la flore donnera l'occasion de rendre plus explicite ces trois niveaux opérants à la classification du règne végétal.

La structure lexicale d'un nom de plante peut être composée d'un terme unique, générique : roz « rose», beuz «buis », linad «ortie», etc. (arbres et plantes courantes). Mais les noms sont le plus communément construits sur le modèle d'un terme de base complété par un déterminant : roz kamm «rose boiteuse», la jonquille ; roz moc'h «rose à cochon» pour le coquelicot ${ }^{5}$, beuz an diaoul ${ }^{6}$ «buis du diable», pour le fragon.

Une étape primordiale dans la description du végétal s'inspire des caractéristiques intrinsèques des plantes et notamment des traits morphologiques. Au rang des premières plantes identifiées se trouvent celles dont on doit se prémunir et qui naissent de réflexes de défense : on décrit celles «qui piquent» par pikerezed «les piqueuses», pour parler du chardon en général, sans discernement entre espèces, ou encore askol pikoù « «askol aux piquants » qui regroupe les cirses, c'est-à-dire le chardon des prés, le chardon de champs voire même le chardon des marais.

4. Claudine FrIEDBERG, «Diversité, ordre et unité dans les savoirs populaires», Natures Sciences, Sociétés, vol. 5, n 1, Paris, 1997.

5. Terme recueilli à Plouaret.

6. Terme recueilli à Pédernec.

7. Dans de nombreux secteurs, le taxon askol réunit à la fois les deux cirses et le genre soncus, autrement dit les laiterons (laiteron maraîcher et laiteron des prés dont différents noms vernaculaires, askol laezh ou askol gwenn, sont souvent confondus et réduits à $a s k o l$ ). 
De nombreuses sociétés perçoivent au moins trois formes dans les végétaux : les herbes, les arbres et les lianes. D'après l'ensemble de mes enquêtes menées en Basse-Bretagne, la catégorie des plantes lianescentes n'apparaît pas identifiée par une appellation distincte. Néanmoins, l'aspect vrillant et torsadé n'échappe pas à l'observateur attentif et revient avec régularité dans les noms, au point de laisser penser que la catégorie "liane» existerait dans la classification populaire mais sans être clairement nommée - ce qu'on appelle une "catégorie implicite» en ethnosciences. Les motivations qui retiennent l'aspect des plantes «qui tournent» incluent le liseron, troell (troiñ : tourner) et le chèvrefeuille, gwezvoud («rameau torsadé $\left.»^{8}\right)$. Le chèvrefeuille est également appelé gwial-gavr («bois flexible à chèvre $»^{9}$ ) : derrière la flexibilité, on retrouve l'attention fixée sur la propension du végétal à tourner. L'assimilation au caprin, en breton comme en français, évoque probablement sa tendance à grimper infatigablement haies et talus : laez gavr (lait de chèvre), boued gavr $^{7}$ (aliment à chèvre)... L'analogie est un principe organisateur récurrent dans les savoirs naturalistes.

Parmi les plantes, on distingue donc les immobiles et celles qui ont la capacité de se mouvoir. Il existe aussi des plantes « qui courent $\gg .$. Le chiendent commun dont les stolons se ramifient et s'étendent vigoureusement fait partie de cette catégorie. Il donne à quiconque a cultivé la terre une impression d'envahissement : localement, il est nommé yeot red, «herbe qui court». Les arbres ou arbustes qui, après leur chute, ont la propriété de marcotter et de repousser vigoureusement sont classés comme koad red, «bois qui court» (saule, noisetier...). Pour la renouée des oiseaux, à la forme rase et tapissante, le «botaniste indigène» utilise également yeot red, à moins qu'il retienne davantage la tige nouée aux multiples

8. Saint-Nicodème, Louargat; du verbe gweañ, tordre, tresser \& bod, rameau; la renouée liseron porte un nom apparenté, gweerez et gwerer selon F. Duros (Fañch Duros, Herbarium vernaculi : lexique du nom des plantes en breton, Quimperlé, La Digitale, 1991).

9. Non issu de la zone d'enquête : Francis FAVEREAU, Geriadur brezhoneg a-vreman, Brezhoneg/galleg galleg/brezhoneg, Dictionnaire du breton contemporain bilingue, Morlaix, Skol Vreizh, 2000. 
segments : louzaouenn mil skloum, "herbe médicinale aux mille nœuds ${ }^{10} \gg$.

La morphologie des plantes offre dans sa multiplicité de nombreuses aspérités, replis et découpes qui accrochent l'esprit herborisateur dans sa rêverie. Les organes multiples et les formes répétées des feuilles, tiges, racines ou fruits offrent la possibilité de repérer des signes propices au dénombrement : pemp ridenn, «cinq nervures» pour le plantain ${ }^{11}$, pemp biz «cinq racines» pour l'œnanthe safranée et la forme de ses racines, mil skloum pour la renouée des oiseaux ${ }^{12}$.

Le même esprit affûté, prêt à traquer le moindre détail du monde végétal ne pouvait pas manquer l'intrigante émission du suc coloré et corrosif qui sort des tiges coupées de la chélidoine, oad belen «sang jaune», de l'euphorbe des bois, laezh bleiz «lait de loup» ou encore des laiterons dénommés askol laezh, laezhegenn, louzaouenn al laez $h^{13}$. L'effet de la couleur s'associe de surcroît aux fascinantes propriétés curatives de la substance pour soigner les verrues chez la chélidoine et l'euphorbe des bois et, ainsi, en faciliter la mémorisation. Dans le camaïeux de la nature, la couleur est incontestablement un élément discriminant pour signifier des différences entre les espèces végétales proches. On rapproche ainsi tout en les opposant deux à deux le spern gwenn et le spern $d u$, respectivement «épine blanche» et «épine noire» (aubépine et prunellier) ${ }^{14}$; dans les terres de landes et de tourbes de Locarn à Saint-Nicodème, les bruyères enrobent les crêtes ventées et les vallons humides de leur couverture

10. Maël Pestivien; également nommée mil skloum al leur à Rospez.

11. Saint-Nicodème, Gourin, Scaër... Nommé pemp gwechad, "cinq veines » à Sainte-Treffine.

12. Le genre des potentilles est repéré dans la botanique populaire pour la fine découpe répétée de ses feuilles : seizh delienn («sept feuilles»: p. ansérine et p. tormentille), pemp delienn (« cinq feuilles » : p. quintefeuille) (F. DuRos, op. cit.).

13. F. Duros, ibid.

14. «Spern melen correspondrait à Rhamnus cathartica (nerprun)» (F. DuRos, ibid.). De nombreuses données hors secteur d'enquêtes pourraient compléter l'énumération des plantes identifiées par les codes de couleur : koad $d u$ «bois noir» pour la bourdaine, louzaouenn velen «herbe médicinale jaune» pour les renoncules, derv $d u$ «chêne noir» pour... le chêne vert, etc. (F. Duros, ibid.) 
mauve. Figurant parmi les éléments les plus familiers de la flore paysanne, on ne sait que trop bien les discerner, d'une part, d'après les nuances de couleur brug $d u$ («bruyère noire» ou cendrée), brug ruz («bruyère rouge» ou ciliée) et brug gwenn («bruyère blanche» ou b. à quatre angles). D'autre part, les caractéristiques du biotope propre à chaque espèce - respectivement les landes xérophiles («sèches»), mésophiles (landes à hygrophilie moyenne) et hygrophiles («humides») - entrent en ligne de compte et facilitent la distinction.

Certains noms relatent la pratique ludique couplée aux propriétés du végétal. Dans un territoire plus large que le secteur d'enquêtes, le chénopode blanc est notoirement connu pour retomber à pic sur ses racines lorsqu' on le lance en l'air. Cela lui vaut son patronyme de plom revr «cul à pic». Une simple observation et un geste anodin en font un savoir remarquablement ancré dans la culture locale, susceptible de déclencher l'hilarité entre les bretonnants amenés à évoquer cette plante. Un savoir-dire la flore qui n'a pas fait l'effet d'une transmission aux jeunes générations, détachées il est vrai de la «chose» botanique. Dans la continuité de cette idée, il n'est plus de mise de s'amuser en éclatant des fleurs de stellaire holostée - dont le nom évocateur «claque» par onomatopée : strakaerien, fleur strak - ou de la digitale strakerezed «éclateuses». Les sens (ici la vue et l'ouie) s'articulent avec le faire.

La motivation de kanel nez, «tige cannelée pour filer la laine» (la grande berce Heracleum sphondylium) provient d'un savoirfaire - la fonction technique du filage de la laine. La motivation est déduite d'une approche expérimentée de la flore. Le nom évoque les cannelures marquées de la tige de la berce qui se substituait avantageusement au fuseau de la quenouille ou du rouet; la tige creuse présente l'intérêt de pouvoir être enfilée le long d'un axe. Le milieu technique influe la perception du milieu naturel pour y transposer une logique analogique. Ici, le toucher interagit avec le faire, l'identification des caractéristiques végétales avec la pratique.

Le fait de qualifier certaines herbes de plantes médicinales provient d'un savoir éprouvé, issu d'une longue connivence au contact du monde végétal, d'une attention aux signes plus vitale que tout autre puisqu'il s'agit de rétablir un équilibre, de maîtriser une défail- 
lance et d'orienter la guérison, derrière laquelle se trame en filigrane le jeu fatal de la vie et la mort. Le terme louzaouenn («herbe médicinale») atteste d'une science empirique populaire et valide des propriétés thérapeutiques fondamentales, celles de soigner hommes et bêtes. Curieusement, peu de noms de plantes nommées louzaouenn ont été collectées au cours des enquêtes, comparativement à ce que la consultation d'ouvrages aurait laissé penser. Deux formes voisines coexistent : soit on met l'accent sur l'organe cible malade (louzaouenn an daoulagad «herbe des yeux» pour la mauve musquée ; louzaouenn an divskouarn « herbe des oreilles» pour la joubarde) soit on souhaite préciser le mal à remédier : louzaouenn poan skouarn «herbe du mal d'oreille» pour la joubarde ou encore louzaouenn an troc' $h$ « herbe à la coupure» pour l'achillée millefeuille.

La littérature regorge de séries de phytonymes qui signalent une vertu thérapeutique. Il est fort probable que ce recours des langues vernaculaires à une terminologie spécialisée intervienne positivement dans l'efficacité curative du végétal. Le remède résonne déjà dans le terme signifiant employé : dire les mots pour combattre les maux, c'est mettre le malade sur la voie - et la voix - de la guérison. L'adoption de règles langagières communes pour nommer rappelle ici combien les soins sont une affaire prise en charge par la collectivité et contribue à la construction sociale de la maladie. Spécifiée par un terme $a d-h o c$, la dénomination fait partie intégrante du dispositif de la thérapie, où entrent en résonance les représentations du mal, du corps, de la plante, des saisons et parfois du milieu naturel. On trouve ici une expression du troisième niveau du processus classificatoire, selon C. Friedberg ${ }^{15}$, organisé par le principe d'unité et de continuité du vivant.

D'autres effets sur l'organisme sont signalés sans spécifier la catégorie louzoù (forme collective de louzaouenn) : stankerez ar gwad «celle qui bouche le sang» (plantain lancéolé et achillée millefeuille) indique leurs propriétés hémostatiques, vitales dans un milieu social habitué à jouer de la faux, de la faucille et autres objets tranchants potentiellement blessants.

15. C. FriedBerg, op. cit. 
Les plantes à usages thérapeutique ou technique renvoient à une flore respectable en raison des services qu'elles rendent. À l'inverse, certains noms de plantes sont connotés péjorativement, notamment associés à l'idée de pauvreté. Vil baourig "vilaine pauvrette» (achillée millefeuille) et paourantez "pauvreté» (bardane) sont porteuses de représentations dévalorisantes. Pour d'autres, résistantes au désherbage et insubordonnées à la loi de la binette, l'accent est mis sur leur tempérament coriace : sous l'identification penn gallet «tête dure», on retrouve l'achillée millefeuille mais aussi le chénopode blanc ${ }^{16}$. Ces plantes dépréciées, classées yeot fall «mauvaises herbes», peuvent également être qualifiées de louzoù, selon les secteurs ${ }^{17}$.

\section{Catégories populaires englobantes}

À l'instar de la catégorie louzoù, il existe une série de taxons populaires englobants qui regroupent des plantes sur des critères de similitude tels que «plante à fleur», «herbe», «fougère», «bois» ou «arbre». Flore souveraine des prés, des talus et bas-côtés, la graminée dite communément yeot «herbe» est un taxon tout aussi prolifique sur le plan lexical qu'il l'est sur le plan biologique. Parmi les nombreux cas, citons yeotenn chapelet «chiendent à chapelet» qui exprime la mise en rapport entre la forme des racines remarquables (agglomérées en une succession de micro-tubercules) et l'objet liturgique : la nomenclature des noms de plantes croise et décroise le monde et pénètre la vie sociale sous de multiples angles. Treuzyeot «l'herbe de travers» est une graminée rebelle, rétive à la maîtrise de la terre par le laboureur, qui pousse à travers champs et jardins grâce à son système de racines traçantes : il s'agit du chiendent commun (également nommé yeot red). Le dactyle aggloméré est appelé yeot gall, «l'herbe française». De la flouve odorante, le nom commun français retient l'enivrant parfum de l'épis, tandis que dans le centre Bretagne c'est l'aspect roussi des extrémités florales que l'on évoque

16. Noté à Minihy-Tréguier.

17. Le terme louzoù est employé sous l'unique acception de plantes indésirables à Plougrescant. Jean LE Dû, Le trégorrois à Plougrescant, Dictionnaire françaisbreton, Morlaix, Emgleo Breiz, 2012. 
à travers yeot rouz. J'ai entendu récemment yeotenn c'hwezh vat, «herbe à la bonne odeur».

La terre armoricaine, terre de fougères, est riche de ses variétés. De manière surprenante, les habitants du pays résument en général la diversité des fougères, indifféremment, sous un même vocable, raden. Pour autant, cela n'empêche pas certains témoins de citer encore les noms d'espèces précises, non sans hésitation ni confusion - ce qui témoigne d'une place résiduelle dans la perception de la flore. La fougère scolopendre, raden dour "fougère d'eau» et la fougère polypode raden puñs, "fougère de puits», sont souvent confondues, probablement du fait de la signification proche de leurs déterminants mais aussi en raison de leur ressemblance anatomique et écologique. La fougère aigle, l'espèce prépondérante, est nommée (par un témoin) raden garzh «fougère des talus».

La catégorie englobante koad «bois» permet de distinguer les espèces de végétaux ligneux. Les espèces d'arbres sont fréquemment distinguées de la sorte : koad kistin «bois de châtaignier», koad der ${ }^{18}$ «bois de chêne», koad vô ${ }^{19}$ «bois de hêtre». Les arbustes sont également précédés par ce qualificatif : koad naered et koad brizh pour la bourdaine («bois de vipère» en raison de ces baies noires et «bois tacheté» pour son écorce ponctuée de tâches blanches); koad skô «bois de sureau»; koad kraoñv garzh, «bois de noix de talus» pour le noisetier etc.

Lorsque l'on souhaite mettre l'accent sur les propriétés spécifiques liées au bois, le terme koad prime dans la conversation. Sinon, on nomme une espèce végétale munie d'un tronc autoportant et de branches, de haute stature, autrement dit un «arbre» par gwez. Chaque espèce est spécifiée : gwez kistin «châtaignier», gwez der «chêne», etc. Dans le secteur d'étude, on appelle rarement un arbre uniquement par son substantif. Par exemple, "châtaignier» ne se traduit pas littérallement par kistin, mais par «arbre-châtaignier», gwez kistin. Le même principe est appliqué pour l'arbuste, appelé bodenn ou bouchenn (employé au singulatif qui signifie littéralement «touffe») : bodenn skô «sureau», bodenn halleg «saule».

18. Selon la prononciation locale.

19. L'orthographe du hêtre est faou en breton écrit et unifié mais très éloigné d'une prononciation courante en centre Bretagne, vô. 
En ce qui concerne la catégorie des «plantes à fleur», on emploie divers substantifs fleur $^{20}$, boked $^{21}$, et parfois bleuñv ${ }^{22}$. Dans le breton usuel actuel, le terme bleuñv désigne le plus souvent les arbres en fleur : l'arrivée du printemps est consacrée par l'expression «Ai eo en bleuñv ar gwez. Les arbres sont en fleur». La fleur amann «fleur de beurre» dévoile non seulement l'analogie entre la couleur du beurre et celle des pétales éclatants de la ficaire, mais la fleur aux pétales jaune-citron rappelle aussi par son aspect solaire le sens prémonitoire de la saison claire et chaude à venir. Son fleurissement au mois de janvier fait scintiller les sous-bois de mille éclats et célèbre les jours qui rallongent. De même, on dit boked laezh «fleur de lait» ou bleuig an hañv "petite fleur de l'été» pour saluer la primevère, annonciatrice du retour attendu de la belle saison et l'espoir d'une fécondité des prés dont résultera, on l'espère, la bonne production du lait. La flore, les saisons et la production laitière : les trois éléments scellés à travers le même signifiant s'insèrent dans le troisième niveau d'organisation du vivant défini par C. Friedberg ${ }^{23}$, système cohérent englobant le fonctionnement d'une société.

\section{Fascinantes liaisons faune-flore}

Le règne animal, amplement convoqué pour nommer les plantes, y compris pour les «plantes à fleur», me permet d'ouvrir un volet sur la fascination populaire pour la complicité entre faune et flore. L'oxalis ou pain de coucou, fleur koukoug «fleur de coucou» (Oxalis acetosella), fleur bran "fleur de corbeau» (jacinthe des bois), fleur viper "fleur de vipère» (arum tacheté), fleur louarn «fleur du renard» (digitale), etc. : les noms établissent des liens symboliques entre les deux règnes et valident l'idée de compréhension totalisante et de continuité des êtres vivants. À titre de comparaison, la pensée scientifique, forgée sur la rupture nature-culture, considérerait

20. Fleur dont le singulatif est fleurenn pour désigner une fleur précise, isolée des autres.

21. On use ici du pluriel bokedou ou bokidi, lorsque l'on met l'accent sur un ensemble de fleurs.

22. Usage également du pluriel bleuñviou.

23. C. FRIEDBERG, op. cit. 
les interrelations faune-flore dans un sous-compartiment nommé «écologie».

La floraison de fleur koukoug est un signe floral pour annoncer le retour du coucou à la même saison. À travers cette mise en résonance du monde animal et du monde végétal, la végétation matérialise le marquage biologique de l'écoulement cyclique du temps. La fleur viper est nommée ainsi en guise d'avertissement pour signaler la toxicité potentielle des plantes à fruits rouge ${ }^{24}$. Toxicité de l'une et venin de l'autre suscitent le rapprochement analogique. L'arum tacheté, aux fruits écarlates en grappes (et non des fleurs; mais on sait que la tradition populaire n'a pas toujours un respect scrupuleux des règles botaniques) marque même un territoire où sévit une vipère, à en croire certains. Marquage du temps, pour l'une, et marquage de l'espace, pour l'autre, témoignent d'une production sociale du temps et de l'espace où se reflètent des objets naturels sauvages, par un jeu de correspondances et de discontinuités.

Qu'elle soit fleur viper ou louzaouenn an naered (arum tacheté), krampouezh koukoug «crêpe du coucou» (nombril de Vénus), etc., ces noms de plantes forment un ensemble qui renforce l'idée d'un lien alimentaire de la plante sauvage au monde animal. L'image du pain, de la crêpe et des herbes médicinales évoque des usages élaborés, électifs et spécifiques qui laissent supposer que les animaux s'alimentent et se soignent avec des plantes précises. Ce principe esquisse le concept de culture (alimentaire et thérapeutique) au sein du règne animal, par analogie à la culture des humains. L'ensemble de ces constructions lexicales fondait-elle les représentations passées

24. Le mécanisme de la phobie opère partout en Basse-Bretagne dans la mise en garde vis-à-vis des fruits à baies (rouges ou noires). Elles sont marquées du sceau de l'interdit, signifié par des dénominations de "plantes à vipère». La bourdaine est parfois nommée «bois de vipère». L'«herbe aux vipères» désigne fréquemment l'arum tacheté (Arum italicum). Le sceau de Salomon est signalé par un dissuasif «fougère à vipère». Pour le chèvrefeuille, $\mathrm{j}$ ' ai recueilli un nom de «fleur à vipère». Catégorie populaire spécifique au sein de la classe des végétaux à déterminant animal, elle a donc des visées dissuasives et «pédagogiques» à destination de l'enfant imprudent. Dans le même ordre d'idées, les champignons sont assimilés au monde des crapauds, appelés «bonnets/chapeaux à crapauds » ou « escabeau à crapauds». Comme toute catégorie, elle est destinée à contribuer à la mise en ordre symbolique du monde naturel. Sur le plan de la classification locale, la baie ne désigne aucunement un fruit mais une «boule». 
d'une communauté animale, comme transposition de la communauté humaine?

Une autre série de noms de plantes est construite par l'ajout d'un déterminant au nom d'animal. Trois couples d'arbres sont ainsi concernés : le cerisier kelez (Prunus cerasus) est opposé au merisier, kelez mouilc'hi, «cerisier à merle»; le poirier considéré sauvage kozhper $^{25}$ "sale-poirier» est doublé d'un «sale-poirier à merle», kozhper mouilc'hi, qui se voit ainsi attribuer un statut de «hypersauvage». La nomenclature compare le châtaignier (Castanea silvatica), kistin, au marronnier d'Inde (Aesculus hippocastanum), kistin moc' $h$, «châtaignier à cochon» et les classe de fait au sein de la même catégorie populaire, du même taxon.

Le système fonctionne également pour des plantes herbacées : kaol «choux» est comparé à kaol moc'h, «choux à cochon» pour nommer le rumex ${ }^{26}$. La grande oseille (Rumex acetosa), trichen, est une des rares plantes sauvages mangées en centre Bretagne. Par opposition à la grande oseille, la petite oseille (Rumex acetosela), plus frêle donc moins digne d'attention, est nommée trichen logod, «l'oseille de souris». Sur le plan des qualités gustatives acides, l'oseille est le pendant de l'oxalis (Oxalis acetosela), trichen gad $^{27}$, «oseille à lièvre», plante qui exprime la même saveur du fait de la présence d'acide oxalique dans les feuilles. Dans le cas présent, les plantes ne se ressemblent pas morphologiquement, elles sont ici rapprochées pour leur effet gustatif, qui «casse la soif» selon les témoins... Le petit pois, piz (Pisum sativum), se voit attribuer deux alter-ego dans le domaine sauvage, chez les vesces (Vicia sp.), piz logod «pois de souris» ou piz moc'h «pois de cochon». L'attention populaire se fixe sur la similitude du pois et des vesces dont les fruits forment des gousses.

25. À noter que les locuteurs rencontrés prononcent koper et n'ont pas connaissance de la signification péjorative de kozhper (le terme est délivré par le dictionnaire de F. Favereau. F. FAVEREAU, op. cit. .).

26. Cela ne signifie pas pour autant que c'est un aliment pour les porcs. Sur l'ensemble des témoignages, un seul témoin m'a déclaré que le rumex servait de nourriture des cochons.

27. Recueilli à Ploumilliau. 
La série possède un dénominateur commun, celui de distinguer entre les plantes domestiquées et/ou à usage alimentaire humain, et les plantes qui leur sont apparentées, mais non ingérables et du bord du sauvage.

Deux plantes fourragères s'inscrivent dans la même logique. Les espèces composées d'une variété fourragère et d'une variété à usage humain sont différenciées sur le plan linguistique. Ainsi les pommes de terre cultivées pour les cochons sont à la fois appelées pato moc' $h$, «pommes de terre à cochon» et pato saovach, «pommes de terre sauvages». La variété de blé noir ancien est composée de deux variétés dont on ne peut pas séparer les semences. La forme peu appréciée, au goût amer, et qui devient prépondérante en quelques années dans les cultures est appelée en français «blé noir de Tartarie» et ed-du saovach, "blé noir sauvage» en breton. Elle fournit soit un fourrage pour les chevaux, soit une alimentation d'ajustement pour les familles pauvres. Pour compléter la série, à Scrignac, le marron d'Inde est appelé kistin saovach, "châtaigne sauvage». Cet amalgame entre le pauvre et l'animal se construit par l'assimilation alimentaire : unité de substance, unité de statut.

La même construction lexicale existe dans d'autres langues mais avec un faible recours au couple "végétal \& déterminant animal». En Bourgogne, C. Crosnier recense 6 plantes sur $165^{28}$. Dans le Val d'Anniviers, la proportion est identique ${ }^{29}$. Dans la Vallée Stura (Alpes italiennes), D. Dore et D. Musset en reportent 2 sur 177 et 3 en italien ${ }^{30}$. En Bretagne, 34 plantes sur 203 sont nommées de la sorte.

On observe le phénomène symétriquement inverse pour nommer des espèces du règne animal qui vivent dans la nature. Ainsi le lézard, kazh raden est le «chat des fougères », l'écureuil, kazh koad, le «chat des bois» et le pic-vert, kazeg koad, «jument des bois», etc.

28. C. Crosnier, La cueillette des savoirs. Les usages du végétal - Morvan/ Bourgogne, Parc naturel du Morvan, 1998.

29. S. BrüschweIller, Plantes et savoirs des Alpes. L'exemple du Val D'Anniviers, Sierre, Monographic, 2008.

30. D. Musset, D. Dore, La Mauve et l'erba bianca; guide méthodologique, Salagon, Musée départemental ethnologique de Haute-Provence, 2006. 
Les espèces végétales et animales familières de la ferme se reflètent dans le miroir de la nature où vivent leurs homologues sauvages. Ces espèces ouvriraient des passerelles symboliques entre les deux mondes et formeraient les liens tacites entre le microcosme du milieu anthropisé et le macrocosme extérieur.

Il est une série voisine à ne pas confondre qui regroupe les noms de plantes inspirés d'organes d'animaux ${ }^{31}$. En centre Bretagne, cette attention s'est arrêtée par exemple sur la morphologie du plantain majeur appelé «langue de bœuf», teod ijen, à la spergulaire des champs «barbe de vache», barbeoc' $h$, ou encore à la linaigrette «cheveux de chat», blev kazh. Cette constante trahit la propriété de l'esprit humain, qui à travers la pensée analogique est constamment à la recherche de correspondances entre les objets, les phénomènes ou les processus, vivants ou non-vivants. Le besoin d'élaborer ces connexions symboliques est une nécessité pour établir un ordre des choses. Dans le cas présent, on s'inspire de noms puisés dans un espace qui est familier et proche de l'humain - la sphère animale pour nommer une sphère qui paraît plus lointaine et étrangère à notre condition, le règne végétal.

\section{Références bibliques}

Révélateur du besoin concomitant d'organisation de la nature et d'organisation du social, les noms issus de la religion catholique rappellent aux communs des mortels la séparation de la Création en deux ordres selon des critères bénéfiques ou maléfiques et dont on en attribue l'origine, respectivement, à Dieu ou au Diable. Ainsi si Dieu a créé le cheval, le Diable a conçu l'âne. Et il en va de même pour la flore : Dieu a fait le chêne, le Diable a fait le houx, etc. La référence au diable peut aussi être motivée par des propriétés intrinsèques à la plante qui rappellent le feu : on note le nom de irvin an diaoul le «navet du Diable» pour le tamier, dont la motivation peut être attribuée à son effet rubéfiant ${ }^{32}$. L'application de la plante sur la peau

31. Il s'agit d'une tendance étendue à de multiples sociétés à travers le monde, lesquelles tissent des analogies entre des organes animaux et des traits repérés chez les végétaux.

32. Nom relevé dans le secteur de Maël-Carhaix et Glomel. Dans le secteur de 
produit une réaction vive, une forte démangeaison qui a marqué les esprits des témoins qui ont pu l'essayer. Autre nom recueilli sur le terrain d'enquêtes, celui déjà cité du fragon beuz an diaoul «buis du diable» auquel on aurait attribué des pouvoirs d'ensorcellement de parcelles là où il était planté ${ }^{33}$. On lui attribue des propriétés à l'exact opposé du buis (et du laurier), plantes des Rameaux en Bretagne. D'autres noms font également référence à la Bible : daoulagad ar werc'hez «les yeux de la vierge» pour le myosotis ou yeotenn ar werc'hez «herbe de la vierge», citée comme le nom de l'herbe de l'oubli...

En guise de conclusion, le questionnement des cadres de l'étude en cours permettra en retour d'en esquisser certaines perspectives. S'attacher à la description de ce qu'on pourrait dénommer la «botanique populaire» bretonne dépasse l'horizon d'explicitation d'une simple nomenclature. Tout d'abord, les phytonymes, signes arbitraires, n'ont pas vocation à recouvrir l'ensemble des représentations collectives et des usages qui définissent le végétal ${ }^{34}$. Alors que le signifié n'est pas systématiquement explicite pour les locuteurs, il est en revanche porteur de toutes les dimensions symboliques et tacites de l'objet végétal. Pour éclairer une dénomination, on veillera à la considérer dans un ensemble plus vaste de signes, de pratiques, de savoirs et de représentations, nourris d'écarts et de correspondances, dont la cohérence globale organise le rapport au monde vivant.

Lannion à Gurunhuel, on dénomme la plante gwir irvinenn, le «sur-navet». Gwir ayant une fonction superlative et irvinenn «navet».

33. Cité à Belle-Isle-en-Terre et Saint-Laurent-de-Bégard.

34. Pour brièvement illustrer ce dont les noms de plantes ne traitent pas mais dont les savoirs matériels et idéels sont porteurs et participent pleinement des représentations du végétal, on peut choisir l'exemple tiré de la tradition orale au sujet de la ronce dont un dicton enseigne : An drezenn daoubenneg ' $\mathrm{ra}$ ul lamm d'al labastenneg. La ronce à deux têtes fait chuter l'étourdi. L'expression met en évidence la propriété de la plante de marcotter ("la ronce à deux têtes») et dans laquelle on peut se prendre les pieds. Chacun a pu expérimenter à ses dépens cet effet piégeur, mais de manière tacite. L'intérêt d'une telle expression est de pointer par le verbe une caractéristique biologique et, partant, d'inscrire l'élément remarquable dans l'imaginaire collectif du groupe social. 
Par ailleurs, la nomenclature reconstituée confine à la fiction : elle est dépeinte ici à la manière d'une nature-morte, comme figée dans un ensemble cosmogonique immuable, d'un pan de la langue bretonne désormais peu usité mais reverdi à l'épreuve du terrain ethnographique. Le puzzle qui n'existe en aucun lieu constituerait une figure utopique; il s'offre à nous telle une manipulation en laboratoire, livrée à la fantaisie du chercheur (lequel aspire malgré tout à une production relativement réaliste). Preuve en est, elle résulte notamment d'un remaniement à partir d'éléments éparpillés sur le territoire, parfois éloignés par des distances physiques importantes.

Le puzzle est tout autant le fruit d'une entreprise uchronique : il n'a dû exister intégralement, en l'état, à aucune période de l'histoire. Une telle recomposition, si elle était tenue pour véritable, serait la représentation d'une société décrite comme un ensemble homogène, dans une immatérialité temporelle, interprétée comme insensible à tout effet de variation - culturelle, économique, historique ${ }^{35} \ldots$ Or, il n'est que de regarder la traversée du $\mathrm{XX}^{\mathrm{e}}$ siècle pour reconnaître que le monde rural paysan a subi des crises traumatiques, aux effets d'acculturation et d'amnésie collective ${ }^{36}$.

L'étude des savoirs naturalistes populaires requiert donc une appréciation de la mutation sociétale, par une jauge anthropologique soucieuse des processus de dynamiques sociales, de substitutions culturelles et donc de changements de paradigme. L'instrumentation sociolinguistique permet, non seulement, nous l'avons vu, d'évaluer la coexistence des registres français et breton au sein de la même nomenclature des noms de plantes, mais aussi, d'explorer l'enchâssement progressif de la science officielle dans les savoirs locaux. En outre, observée "à la loupe» en coupes fines, la botanique indigène peut révéler les effets du basculement de la pensée «analogique»

35. Ces propos rejoignent la réflexion d'auteurs comme A. Bensa qui interrogent les dérives d'une anthropologie qui «se laisse souvent bercer par le rêve exotique. En projetant ses présupposés théoriques sur un Autre fantasmé, elle procède à une magnifique fossilisation du temps, de la parole, des cultures et finalement des personnes». Alban Bensa, La Fin de l'exotisme. Essai d'anthropologie critique, Toulouse, Anacharsis, 2006.

36. Laurent GALL, «Une amnésie collective : déni et régression des savoirs populaires botaniques en centre Bretagne», actes du colloque «Traces du Végétal», Angers, 2012 (en cours de publication). 
pré-scientifique vers la pensée «naturaliste » contemporaine de la «modernité ${ }^{37}$.

La tentative de reconstitution - fragmentaire - de la nomenclature des noms de plantes doit être comprise comme un effort de modélisation d'un univers social bien plus complexe, aux multiples paramètres, doublés de fortes variations spatiales et temporelles. Nécessairement réductionniste, borné par des limites arbitraires, le modèle reste néanmoins un objet didactique permettant d'appréhender le processus classificatoire, et, par extension, la conception vernaculaire de l'organisation du vivant.

37. Philippe Descola, Par-delà nature et culture, Paris, Gallimard, Bibliothèque des sciences humaines, 2005. 